\title{
Continuous Ethanol Fermentation at Very High Gravity in the Presence of Saccharomyces cerevisiae: A Bifurcation Analysis
}

\author{
Ghanem-Lakhal Abdelghani \\ Département de Génie des Procédés, ENPC, Constantine, Algérie \\ Email: ghabdelghani@yahoo.com
}

How to cite this paper: Abdelghani, G.-L. (2018) Continuous Ethanol Fermentation at Very High Gravity in the Presence of Saccharomyces cerevisiae: A Bifurcation Analysis. Journal of Sustainable Bioenergy Systems, 8, 116-126.

https://doi.org/10.4236/jsbs.2018.84009

Received: January 14, 2018

Accepted: December 18, 2018

Published: December 21, 2018

Copyright $\odot 2018$ by author and Scientific Research Publishing Inc. This work is licensed under the Creative Commons Attribution International License (CC BY 4.0).

http://creativecommons.org/licenses/by/4.0/

\section{Open Access}

\begin{abstract}
In this paper, a study of the bifurcation analysis of fermentation of sugar to ethanol in presence of Saccharomyces cerivisiae at very high gravity is described. The bifurcation analysis was done for a concentration $280 \mathrm{gl}^{-1}$ of sugar, and the dilution rate was taken as the parameter of bifurcation. Two Hopf bifurcations (HB) at $280 \mathrm{gl}^{-1}$ were found. At dilution rate of $0.027 \mathrm{~h}^{-1}$ the system exhibits damped oscillations and not sustained oscillations as previously reported because the system is close to a point of attraction, and we can attenuate these oscillations by the choice of initial conditions. The system exhibits sustained oscillations between the two Hopf Bifurcations, the first at $0.08028 \mathrm{~h}^{-1}$ and the second at $0.04395 \mathrm{~h}^{-1}$. These oscillations are the consequence of synchrony between the daughter and the mother yeast. Indeed, it is better to take a dilution rate between the two Hopf bifurcations (self sustained oscillations), in order to increase the ethanol productivity.
\end{abstract}

\section{Keywords}

Bifurcation, Ethanol, Fermentation, Saccharomyces cerevisiae, Very High Gravity

\section{Introduction}

The increase of $\mathrm{CO}_{2}$ concentration in the atmosphere, with the possibility of energy deficit, led to an orientation toward biofuels. Bioethanol, being one of the most promising biofuels, is ecologically clean, and has the potential to greatly reduce the dependence to oil. As reported by [1], in Brazil, 50\% of existing cars can use $100 \%$ of ethanol.

Because it is simple and efficient, batch fermentation is currently the predominant process for ethanol production. On the other hand, the continuous ap- 
proaches have some advantages with respect to fermentation. However, for mixtures having a lower ethanol concentration, the product must be distilled [2].

Very high gravity (VHG) fermentation has been thoroughly studied in [3] where it has been shown that although it increases ethanol concentration at the reactor exit and decreases energy consumption for ethanol distillation, some counter effects are induced both at the initial and final phases of the process. At the initial growth phase, the high glucose concentrations produce additional osmotic stress on the yeast cells. On the other hand, at the end of the process, the very high final ethanol concentration inhibits yeast survival, giving a loss of cell viability. When ethanol concentration is over $40 \mathrm{gl}^{-1}$, it inhibits the yeast metabolism and when it exceeds $90 \mathrm{gl}^{-1}$ it becomes toxic [4].

Clearly, these effects should be taken into consideration in the design of efficient processes for bioethanol production because they can act negatively on ethanol production [4].

The ethanol production by fermentation is realized in the presence of the yeast baker Saccharomyce cerevisiae. For continuous fermentation process, oscillations are observed that are difficult to eliminate. Typically, these appear for dilution rates between 0.09 and $0.25 \mathrm{~h}^{-1}$ [5]. Several authors have reported the apparition of sustained oscillations, when the fermentation is realized in continuous culture [6] [7] [8].

In order to avoid or to eliminate these oscillations [9] [10] [11], it is necessary to study the stability of the system. Generally, this is done by a method of bifurcation analysis. Sidhu et al. (2010) [1] have studied the bifurcation of the fermentation in the presence of Saccharomyces cerevisiae, with a low to medium concentration of initial sugar.

In this work, the bifurcation analysis of fermentation of sugar to ethanol in presence of Saccharomyces cerivisiae at very high gravity is presented.

The mathematical model for the fermentation in continuous bioreactor and the kinetic of the bioreaction, and all the constants are taken from Fengwu Bai thesis [3]. The bifurcation analysis was done for concentration $280 \mathrm{gl}^{-1}$. The dilution rate was taken as the parameter of bifurcation, and the results are present in the form of diagram of bifurcation for the different variables of the system.

\section{The Cell Cycle}

In the fermentation process with Saccharomyces cerevisiae two cells, a small one called the daughter and a large one, the mother, are formed at division stage [12]. A population of cells is perfectly synchronized if all cells pass through the same point of their reproductive cycle simultaneously. On the other hand, it is asynchronous if the percentage of cells in each phase of cycle remains constant with time [12].

Because of their small size, daughter cells cannot enter into the division process after separation, only mother cells are able to bud [13]. At each oscillation, the cells that divide do it at the same time. The population is thus partially 
synchronized. In an asynchronously growing population, there is asynchrony in stage G1 (the first of four phases of the cell cycle) growth time requirement among daughter's cells. Indeed, the daughter cell sizes at completion of cell division vary, and are smaller than the critical size requirement (CSR). In contrast, mother cell sizes are more nearly equal to the CSR [14]. In stage G2 (the phase preceding mitosis), a bud emerges and grows until cell division occurs. In [14], it was observed that duration of G2 is much less sensitive to various parameters of growth than stage G1 and is relatively constant (synchronous) in the asynchronously growing population.

\section{Ethanol Effect}

At high feed glucose concentrations, high inhibitory ethanol concentrations are observed only under VHG conditions. Ethanol concentrations over $40 \mathrm{gl}^{-1}$ exert inhibition on yeast metabolism, and turns to toxicity if there is an excess in ethanol concentration up to about a $90 \mathrm{gl}^{-1}$ [4].

Cells in high ethanol concentration may either die or lose irreversibly their ability to divide. In the latter case, they can still produce ethanol since the fermentation remains active [15].

The stress due to either yeast growth or ethanol production yields certain metabolites such as Glycerol and trehalose. At the same, time ATP (Adenosine triphosphate) is produced to satisfy the increased energy demands of the cell under stress.

According to the observations of Ding [16], in response to the destruction of the normal membrane structure caused by ethanol increase, yeast cells may change membrane composition.

\section{Model Description [3]}

The model of Li et al. for the oscillations observed in continuous ethanol fermentation with $\mathrm{Z}$. mobilis is based on the ethanol concentration change rate history [17].

Since the ethanol concentration history is more significant than the ethanol concentration change rate history, Bai [3] has modeled the lag response of yeast cells to ethanol concentration history by introducing a weights function: a dynamic specific growth rate and a dynamic specific ethanol production rate, because ethanol production is tightly associated with the growth of yeast cells. The ethanol concentration which happened before has most significant influence on current status. The model indicated that there is a delayed response of yeast cells to ethanol inhibition effect, since the yeast cells do not respond immediately to the ethanol inhibition exerted on them, but require a period of time to adjust their intracellular metabolism to adapt such an ethanol inhibition environment.

The Equations of the proposed model are [3]: (Table 1)

$$
\frac{\mathrm{d} X}{\mathrm{~d} t}=X(Z-D)
$$


Table 1. Parameters used for the model [3].

\begin{tabular}{cc}
\hline Parameter & Value \\
\hline$D$ & 0.027 \\
$M u_{\max }$ & 0.40 \\
$K_{s}$ & 10.8 \\
$K_{i}$ & 293.2 \\
$K_{s p}$ & 23.0 \\
$K_{i p}$ & 1751.7 \\
$\alpha$ & 3.86 \\
$\beta$ & 1.66 \\
$P_{m}$ & 167 \\
$\mu_{0}$ & $0\left(\right.$ if $\left.S>K_{S}\right)$ \\
& $0.0378\left(\right.$ if $\left.S \leq K_{S}\right)$ \\
$v_{0}$ & $0\left(\right.$ if $\left.S>2 K_{S P}\right)$ \\
$v_{\max }$ & $0.246\left(\right.$ if $\left.S \leq 2 K_{S P}\right)$ \\
$\omega$ & 1.66335 \\
$Y_{p s}$ & 0.0617 \\
& 0.45 \\
\hline
\end{tabular}

$$
\begin{aligned}
\frac{\mathrm{d} S}{\mathrm{~d} t}= & D\left(S_{0}-S\right)-\frac{1}{Y_{P / S}} Z p X \\
\frac{\mathrm{d} P}{\mathrm{~d} t} & =-D P+Z p X \\
\frac{\mathrm{d} Z}{\mathrm{~d} t} & =\omega\left(V_{G}-Z\right) \\
\frac{\mathrm{d} V_{G}}{\mathrm{~d} t} & =\omega\left(\mu-V_{G}\right) \\
\frac{\mathrm{d} Z p}{\mathrm{~d} t} & =\omega\left(V_{P}-Z p\right) \\
\frac{\mathrm{d} V_{P}}{\mathrm{~d} t} & =\omega\left(v-V_{P}\right)
\end{aligned}
$$

In the kinetic model the specific growth rate is expressed into two terms. One non linear term for the ethanol concentration, and another term for the substrate inhibition with the introduction of substrate constant into the kinetic expressions [3]. Bai has added an experimental parameter $\mu_{0}$ that equals the dilution rate at which the limiting substrate concentration falls below a detection limit.

$$
\mu=\mu_{\max } \frac{S}{K_{S}+S+S^{2} / K_{i}}\left(1-\frac{P}{P_{\max }}\right)^{\alpha}+\mu_{0}
$$


Since the growth of cells depends tightly on ethanol production, the following similar model was proposed [3]:

$$
v=v_{\max } \frac{S}{K_{S P}+S+S^{2} / K_{i P}}\left(1-\frac{P}{P_{\max }}\right)^{\beta}+v_{0}
$$

\section{Results and Discussion}

Numerical results were obtained using the software XPPAUT Version 6.10 [18]. XPPAUT can solve ordinary differential equations, and make a bifurcation analysis with an incorporated bifurcation software AUTO. The dilution rate $D$ was taken as the bifurcation parameter, and the bifurcation analysis was done for a feed concentration of sugar at $\mathrm{CS} 0=280 \mathrm{gl}^{-1}$.

Figure 2 represents the bifurcation diagrams for the ethanol concentration. As we decrease the inlet dilution rate, we can observe different stages in the fermentation. In the first region, the system exhibits a unique stable steady-state.

As the dilution rate is decreased, we observed a second region, in which a first supercritical Hopf bifurcation HB1 occurs with $D=0.08028 \mathrm{~h}^{-1}$. This region contains a unique stable periodic branch, represented black bold line in Figure 1, giving an apparition of stable limit cycle with sustained oscillations, represented by the red circle in Figure 1. In the case synchrony of cell growth, modulation of the metabolism appears clearly on the reactor the measured variables. For example, at dilution rate $0.07 \mathrm{~h}^{-1}$, taken between the two Hopf bifurcations, the system exhibits sustained oscillations as consequence of the synchrony of the cycle, and there is an increase of the productivity over the steady state, as shown in Figure 2.

In the case synchrony of cell growth, modulation of the metabolism appears clearly on the reactor the measured variables.

With the decrease of the dilution rate a second Hopf bifurcation HB2 occurs at $D=0.04395 \mathrm{~h}^{-1}$ where the limit cycle disappears, and a stable steady state is created. This is represented by the black bold line in Figure 1.

At dilution rate $0.027 \mathrm{~h}^{-1}$ and for initial concentration of sugar $280 \mathrm{gl}^{-1}$, as shown in Figure 3, there is steady state where the oscillations are damped. The cell synchrony decay may be the result of the random change of the time required for cell mitosis. This produces increase cell population heterogeneity [19].

An asynchronous step may be made synchronous in at least two ways [14]. Cells may be allowed to complete an asynchronous step during arrest at a block, resulting in the elimination of the asynchronous step from the cell cycle for one or more generation after releasing the block. Second, creating conditions which accelerate the rate of completion of an asynchronous step is equivalent to making that step more synchronous. At a rate of $0.027 \mathrm{~h}^{-1}$ all cells start dividing synchronously for several generations until the loss of synchrony.

Shen [10] reported that the fermentation system was initiated at the dilution rate of $0.04 \mathrm{~h}^{-1}$ and run continuously until the steady state was established. The 


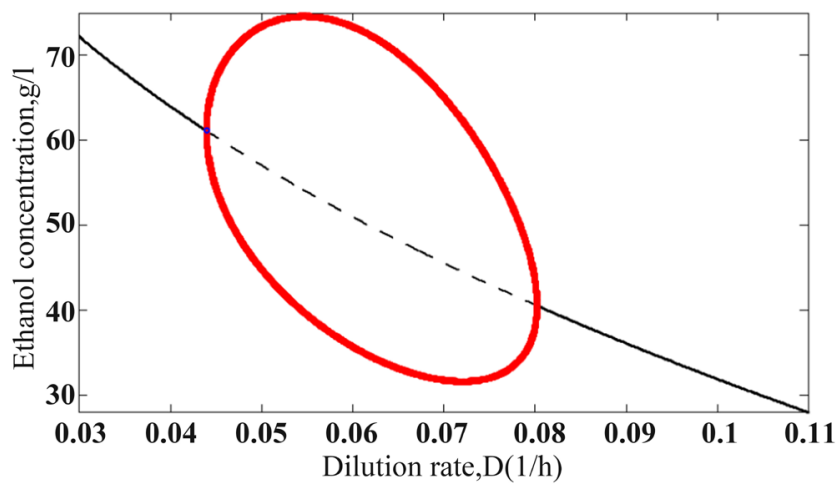

(a)

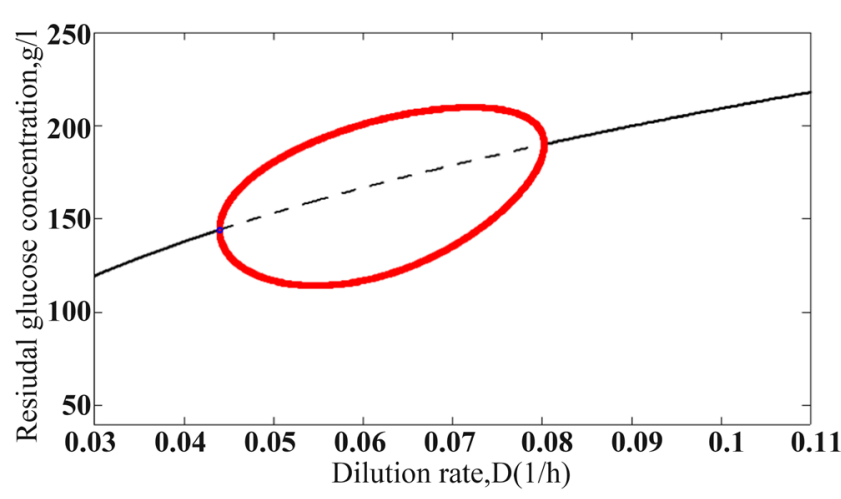

(b)

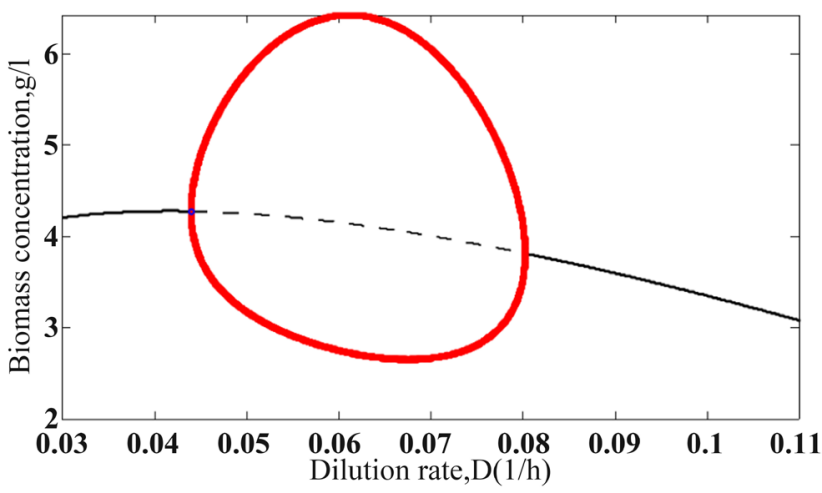

(c)

Figure 1. Bifurcation diagrams with dilution rate D as the bifurcation parameter and $\mathrm{CS} 0=280 \mathrm{gl}^{-1}$, periodic branch ( $\longrightarrow$ ), stable steady-state branch (-), unstable steady-state branch (---). (a) ethanol concentration, (b) residual substrate concentration, (c) biomass concentrate.

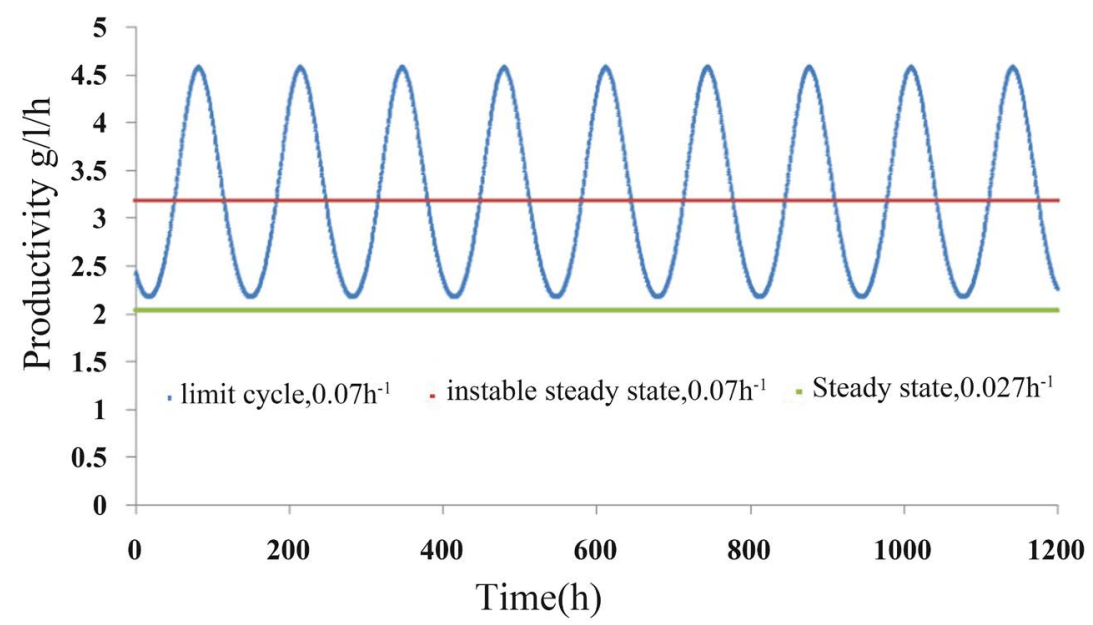

Figure 2. Dynamic simulations of the Productivity for CSO $=280 \mathrm{gl}^{-1}, \mathrm{D}=0.07 \mathrm{~h}^{-1}$, and final time $t_{f}=1200 \mathrm{~h}$.

dilution rate was then switched to $0.027 \mathrm{~h}^{-1}$ for another steady state. Oscillations were introduced when the dilution rate was switched back to $0.04 \mathrm{~h}^{-1}$ and the fermentation system was maintained at the oscillatory state for five periods, about 4 weeks. This indicates that there are two steady states, one at $0.027 \mathrm{~h}^{-1}$ 


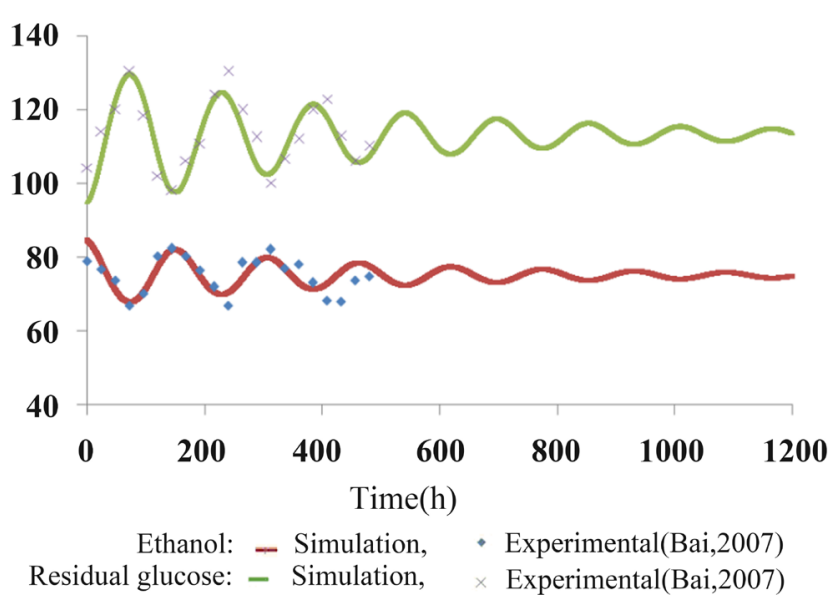

(a)

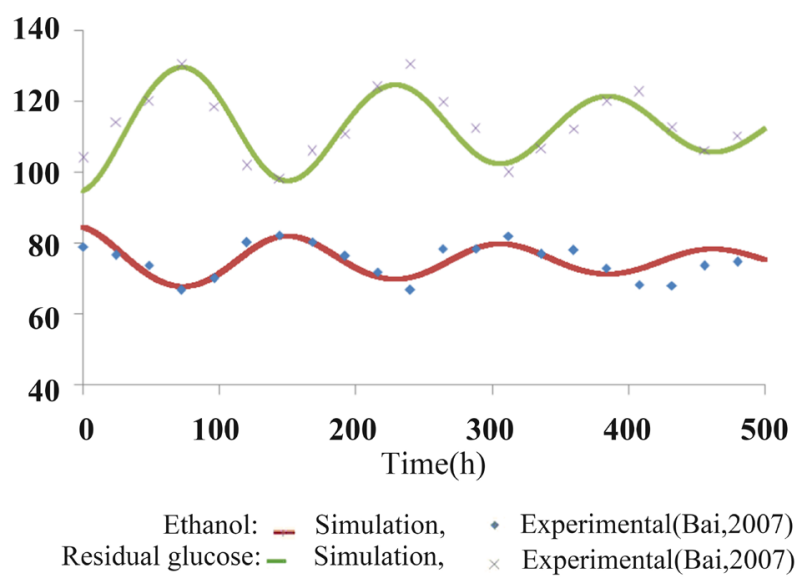

(b)

Figure 3. (a) Dynamic simulations of the oscillations of residual glucose, ethanol for CS0 $=280 \mathrm{gl}^{-1}, D=0.027 \mathrm{~h}^{-1}$, and final time $t_{f}=1200 \mathrm{~h}$; (b) Dynamic simulations of the oscillations of residual glucose, ethanol for $\operatorname{CS} 0=280 \mathrm{gl}^{-1}, D=0.027 \mathrm{~h}^{-1}$, and final time $t_{f}=$ $480 \mathrm{~h}$.

and another at $0.04 \mathrm{~h}^{-1}$. Therefore, the oscillations are damped, as we can see on Figure 4(a). Indeed, while increasing the time for the fermentation process, from $480 \mathrm{~h}$ to $1200 \mathrm{~h}$ as shown in Figure 3(a), it appears that the oscillations are effectively damped, as predicted by bifurcation analysis.

If the initial conditions for the fermentation do not correspond to the steady state, the system oscillates around the steady state and will converge to it over the time. This phenomenon is due to the change in the time required for cell division that causes a decay of cell synchrony as stated before [19].

When we are far from the steady state, it will take a long time to reach it, and the oscillations seem to be quasi sustained. Figure 4(a) shows that if we are near the steady state, the system converges to it faster and this becomes visible on experimental concentrations. For $D$ equals $0.04 \mathrm{~h}^{-1}$ and for moderate time the oscillations seems to be sustained (quasi limit cycle), but if the time is too long the oscillations are damped (a steady state), this is apparent in Figure 4 (c). 


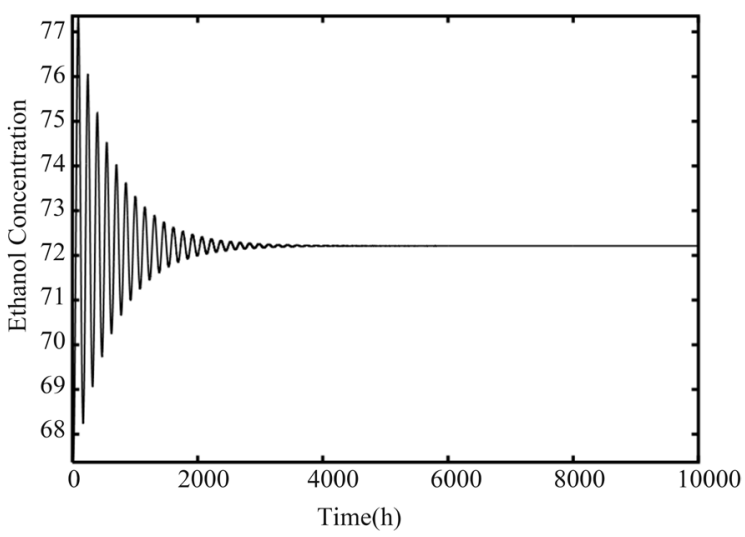

(a)

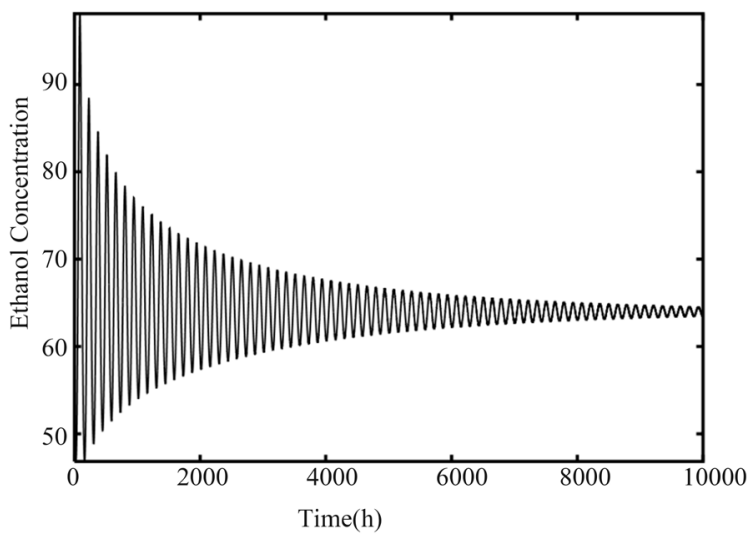

(c)

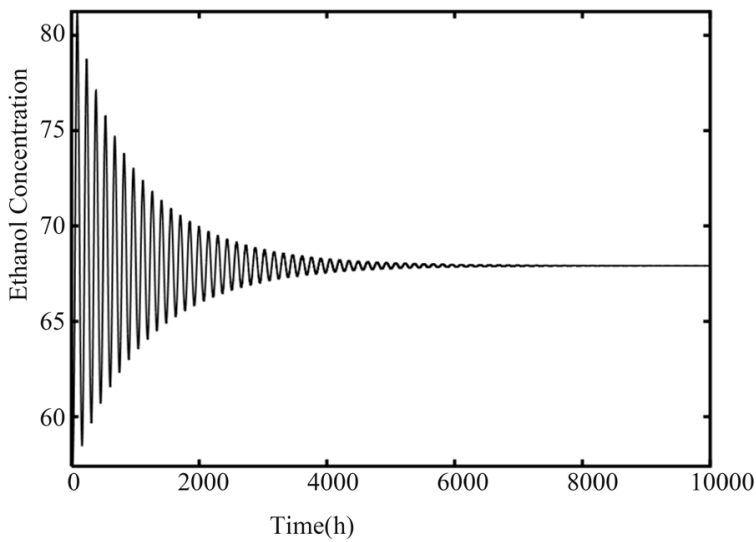

(b)

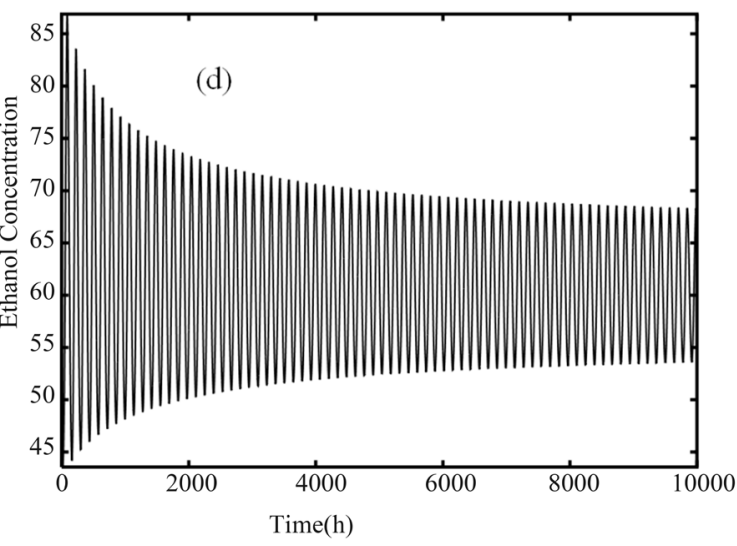

(d)

Figure 4. Dynamic simulations of the oscillations of ethanol for CS0 $=280 \mathrm{gl}^{-1}$, and final time $t_{f}=10000 \mathrm{~h}$, (a) $D=$ $0.03 \mathrm{~h}^{-1}$, (b) $D=0.035 \mathrm{~h}^{-1}$, (c) $D=0.04 \mathrm{~h}^{-1}$, (d) $D=0.045 \mathrm{~h}^{-1}$.

At low dilution rate the oscillations are damped, and with the increase of the dilution rate, the behavior is more and more synchronous and the oscillations tend to be sustained, as shown in Figure 4(d).

\section{Conclusions}

In this paper, the bifurcation analysis of the fermentation of sugar into an ethanol in the presence of yeast Saccharomyces cerivisiae was studied. It was found that the system stability is dependent on dilution rate and on the initial concentration of sugar.

At initial concentration of sugar equal $280 \mathrm{gl}^{-1}$, the system exhibits two Hopf bifurcations at high dilution rate. The first HB is located at $0.04395 \mathrm{~h}^{-1}$ and the second at $0.08028 \mathrm{~h}^{-1}$. The system presents a sustained oscillation between the Hopf bifurcations as shown by the red circle in Figure 1. This phenomenon is reported by several authors [6] [7] [8], and is mainly caused by ethanol concentration and the synchrony of cell cycle of the mother and daughter cells. Indeed, a steady state is caused by the asynchrony of cell cycle, and the limit cycle is caused by the synchrony of the cell cycle [20]. 
At dilution rate $0.027 \mathrm{~h}^{-1}$, there is a steady state where the oscillations are damped, and a not quasi-steady sate, with a sustained oscillations as reported by [3] [21], which correspond to a limit cycle which is shown by red circle in Figure 1.

As a consequence, we can attenuate or eliminate the oscillations simply by choosing initial concentrations corresponding to the steady state. The ethanol concentration influences directly the production by influencing the viability of the cells Indeed, the ethanol concentrations over $40 \mathrm{gl}^{-1}$ exert inhibition on yeast metabolism, and turns to toxicity when ethanol concentrations in the fermentation broth exceed $90 \mathrm{gl}^{-1}$ [4], and it influences the production of ethanol indirectly by influencing the synchrony of the cell cycle.

\section{Conflicts of Interest}

The author declares no conflicts of interest regarding the publication of this paper.

\section{References}

[1] Sidhu, H.S., Watt, S.D., Nelson, M.I. and Ray, A.K. (2010) Analysis of a Model for Ethanol Production through Continuous Fermentation: Ethanol Productivity. International Journal of Chemical Reactor Engineering, 8, 1-17.

[2] Bai, F.W., Anderson, W.A. and Moo-Young, M. (2008) Ethanol Fermentation Technologies from Sugar and Starch Feedstocks. Biotechnology Advances, 26, 89-105. https://doi.org/10.1016/j.biotechadv.2007.09.002

[3] Bai, F.W. (2007) Process Oscillations in Continuous Ethanol Fermentation with Saccharomyces cerevisiae. PhD Thesis Waterloo. http://hdl.handle.net/10012/2771

[4] Srinivasan, S. (2012) Effect of Dissolved Carbon Dioxide on Very-High-Gravity Fermentation. Master of Science, University of Saskatchewan, Saskatoon.

[5] Mhaskar, P., Henson, M.A. and Hjortso, M.A. (2002) Cell Population Modeling and Parameter Estimation of Continuous Cultures of Saccharomyces cerevisiae. Biotechnology Progress, 8, 1010-1026. https://doi.org/10.1021/bp020083i

[6] Skupin, P. and Metzger, M. (2015) Oscillatory Behavior Control in Continuous Fermentation Processes. IFAC-PapersOnLine, 48, 1114-1119.

https://doi.org/10.1016/j.ifacol.2015.09.117

[7] Patnaik, P.R. (2003) Oscillatory Metabolism of Saccharomyces cerevisiae: An Overview of Mechanisms and Models. Biotechnology Advances, 21, 183-192. https://doi.org/10.1016/S0734-9750(03)00022-3

[8] Chen, C.I. and McDonald, K.A. (1990) Oscillatory Behavior of Saccharomyces cerevisiae in Continuous Culture, I and II. Biotechnology Progress, 36, 28-38.

[9] Garhyan, P., Elnashaie, S.S.E.H., Al-Haddad, S.M., Ibrahim, G. and Elshishini, S.S. (2003) Exploration and Exploitation of Bifurcation/Chaotic Behavior of a Continuous Fermentor for the Production of Ethanol. Chemical Engineering Science, 58, 1479-1496. https://doi.org/10.1016/S0009-2509(02)00681-4

[10] Shen, Y., Ge, X.M. and Bai, F.W. (2010) Application of Oscillation for Efficiency Improvement of Continuous Ethanol Fermentation with Saccharomyces cerevisiae under Very-High-Gravity Conditions. Applied Microbiology and Biotechnology, 
86, 103-108. https://doi.org/10.1007/s00253-009-2283-2

[11] Ghanem-Lakhal, A. (2017) Bifurcation Analysis of Ethanol Fermentation at Very High Gravity. Dignified Researchers in Agricultural, Biological \& Life Sciences (DRABL) Conferences, Istanbul, 17-18 May 2017.

[12] Priori, L. and Ubezio, P. (1996) Mathematical Modelling and Computer Simulation of Cell Synchrony. Methods in Cell Science, 18, 83-91. https://doi.org/10.1007/BF00122158

[13] Duboc, P., Marison, I. and Stockar, U. (1996) Physiology of Saccharomyces cerevisiae during Cell Cycle Oscillations. Journal of Biotechnology, 51, 57-72. https://doi.org/10.1016/0168-1656(96)01566-0

[14] Moore, S. (1984) Synchronous Cell Growth Occurs upon Synchronizing the Two Regulatory Steps of the Saccharomyces cerevisiae Cell Cycle. Experimental Cell Research, 151, 542-556. https://doi.org/10.1016/0014-4827(84)90402-6

[15] Ghommidh, C., Vaija, J., Bolarinwa, S. and Navarro, J.M. (1989) Oscillatory Behavior of Zymomonas mobilis in Continuous Cultures, a Simple Stochastic Model Biotechnology Letters, 2, 659-664. https://doi.org/10.1007/BF01025278

[16] Ding, J., Huang, X., Zhang, L., Zhao, N., Yang, D. and Zhang, K. (2009) Tolerance and Stress Response to Ethanol in the Yeast Saccharomyces cerevisiae. Applied Microbiology and Biotechnology, 85, 253-563. https://doi.org/10.1007/s00253-009-2223-1

[17] Li, J., McLellan, P.J. and Daugulis, A.J. (1995) Inhibition Effects of Ethanol Concentration History and Ethanol Concentration Change Rate on Zymomonas mobilis. Biotechnology Letters, 17, 321-326. https://doi.org/10.1007/BF01190646

[18] B. Ermentrout, http://www.mathematics.pitt.edu/ bard/xpp/xpp.html

[19] Bailey, K.M., Venkatasubramanian, K. and Karkare, S.B. (1985) Immobilized Live Cell Reactor Dynamics Following Dilution Rate Shift to Growth Conditions: Cell Synchrony Effects. Biotechnology and Bioengineering, 27, 1208-1213. https://doi.org/10.1002/bit.260270816

[20] Henson, M.A. (2003) Dynamic Modeling and Control of Yeast Cell Populations in Continuous Biochemical Reactors. Computer and Chemical Engineering, 27, 1185-1199. https://doi.org/10.1016/S0098-1354(03)00046-2

[21] Bai, F.W., Chen, L.J., Anderson, W.A. and Moo-Young, M. (2004) Parameter Oscillations in Very High Gravity Medium Continuous Ethanol Fermentation and Their Attenuation on Multi-Stage Packed Column Bioreactor System. Biotechnology and Bioengineering, 88, 558-566. https://doi.org/10.1002/bit.20221 


\section{Nomenclature}

$D$ : dilution rate, $\mathrm{h}^{-1}$

$K_{S}$ : intrinsic Monod constant for growth, $\mathrm{gl}^{-1}$

$K_{i}$ intrinsic substrate inhibition constant for growth, $\mathrm{gl}^{-1}$

$K_{s p}$ : observed Monod constant for growth, $\mathrm{gl}^{-1}$

$K_{i p}$ : observed substrate inhibition constant for growth, $\mathrm{gl}^{-1}$

$P$. Ethanol concentration, $\mathrm{g}^{-1}$

$P_{\max }$ : maximum ethanol concentration for growth and ethanol formation, $\mathrm{gl}^{-1}$

$S:$ residual glucose concentration, $\mathrm{gl}^{-1}$

$S_{0}$ : Input glucose concentration, $\mathrm{gl}^{-1}$

$X$ : biomass concentration, $\mathrm{gl}^{-1}$

$\mu$ : intrinsic specific growth rate, $\mathrm{h}^{-1}$

$\mu_{0}$ : specific growth rate at lower dilution rates for lower gravity medium, $\mathrm{h}^{-1}$ $\mu$ max: intrinsic maximum specific growth rate, $\mathrm{h}^{-1}$

$v$ : intrinsic specific ethanol production rate, $\mathrm{h}^{-1}$

$v_{0}$ : specific ethanol production rate at lower dilution rates for lower gravity medium, $\mathrm{h}^{-1}$

$\alpha$ : ethanol inhibition constant for growth

$\beta$ : ethanol inhibition for ethanol formation 